\title{
Aplicação de um modelo paramétrico multivariado para o controle da temperatura de fornos de túnel
}

\author{
Adriano Mendonça Souza \\ Coordenador do Curso de Especialização em Estatística e Modelagem Quantitativa - UFSC \\ E-mail:amsouza@ccne.ufsm.br \\ Robert Wayne Samohyl \\ Professor do Programa Pós-graduação em Engenharia de Produção e Sistemas - EPS - UFSC \\ E-mail: samohyl@eps.ufsc.br \\ César O. Malavé \\ Associate Professor Texas A \& M University. Department of Industrial Engineering \\ E-mail:malave@tamu.edu
}

\begin{abstract}
Resumo
O principal objetivo desta pesquisa é implementar um ajuste multivariado proporcional, em relação ao último erro, das variáveis que estão fora do alvo desejado. Para a realização do ajuste multivariado proporcional, utilizou-se um forno de túnel para a queima de azulejo. Dessa forma, foi possível analisar todas as etapas do processo produtivo, para efetivar a equação de controle proposta. As etapas seguidas foram: a aplicação da estatística de médias móveis ponderadas exponencialmente, que determinará o comportamento dos distúrbios; os valores futuros das variáveis serão estimados por regressões aparentemente não correlacionadas, pois existe relação entre as variáveis e entre os erros das equações estimadas; e aplicação da equação de controle proposta. Dessa forma, um ajuste de realimentação multivariado pode ser alcançado de modo científico, controlando, assim, a temperatura do forno.
\end{abstract}

\section{Palavras-chave}

Controle de realimentação multivariado; regressões aparentemente não correlacionadas, controle de engenharia do processo.

\section{A multivariate application of parametric model to control the tunel oven temperature}

\begin{abstract}
The main purpose of this research is to implement a multivariate feedback adjustment proportional to the last deviation in relation to the target of the variables that wandering around the target. In order to apply the controller equation it will be necessary to study the exponentially weighted moving average statistics, so that the target disturbances behavior is determined. In order to determine the future values of the variables seemingly unrelated regression is used, once there is a relation among the variables and among the errors of the estimated equations. To apply this methodology, a tunnel oven to burn tile was used, so this way a multivariate feedback adjustment can be reached in a scientific way, controlling the oven temperature.
\end{abstract}

Key words

Multivariate feedback control, seemingly unrelated regression, engineering process control. 


\section{INTRODUĈ̣̃O}

Os sistemas de controle de realimentação são amplamente usados em quase todos os aspectos da vida moderna. Para Franklin et al. (1994), um sistema de realimentação é o processo de medição da variável de entrada, a qual é usada para influenciar o valor da variável de saída, onde, estas, servem para informar como se deve manipular as variáveis de entrada.

Como as variáveis que influenciam o processo devem ser reguladas através de estimativas, defende-se a idéia de que um bom controlador deve ter o processo de estimação separado do processo de controle (ASTROM; WITTENMARK, 1989), uma vez que, na maioria dos controladores, existe um algoritmo de estimação que fornece os parâmetros estimados para serem utilizados no controlador. A equação de controle utiliza essas estimativas, feitas do processo, efetivando os ajustes necessários. Se a estimação for feita, independentemente da ação de controle, é possível fazê-la fora da linha de produção e, sempre que se tiver um conhecimento prévio das variáveis, pode-se decidir quais restrições devem ser impostas nas variáveis de controle.

Pesquisadores, como Box et al. (1978), Mac Gregor (1987), Box \& Kramer (1992), Ramirez (1994), Del Castillo (1996), Box \& Luceño (1997), têm apresentado diversos estudos e formas de como o ajuste de realimentação pode ser feito, utilizando um conjunto de dados históricos, que possibilita o conhecimento do comportamento futuro da série.

O objetivo desta pesquisa é implementar um controlador proporcional ao erro, em um conjunto de variáveis multivariadas, que apresenta diversas características a serem monitoradas e/ou realimentadas e que, na maioria das vezes, são tratadas como variáveis independentes. Para se atingir esse objetivo maior, será necessário fazer a estimação simultânea das equações de regressões, determinar a constante de ponderação dos erros, a constante de impacto, que é a influência que a alteração realizada em uma unidade da variável de entrada causará no sistema e, principalmente, fazer uma avaliação da estabilidade do mesmo.

Os controladores atuam para regular o processo, agindo diretamente nas variáveis que irão influenciar nas características finais do produto, tais como: temperatura, pressão do fluxo de corrente e tempo que deve permanecer em cada estágio do processo, ou a cada mudança que houver.

Para atingir as características finais do produto, uma equação de realimentação foi estabelecida, utilizando-se os parâmetros encontrados através de uma metodologia que leva em consideração o relacionamento das variáveis, proporcionando uma melhor precisão nas estimativas dos mesmos.

A contribuição desta pesquisa não se deve, somente, à realização da equação de controle, mas ao fato de que este controlador leva em consideração múltiplas variáveis a serem controladas, e que, na maioria dos trabalhos encontrados na literatura, descrevem-se trabalhos univariados que utilizam, apenas, equações de primeira ordem, e, aqui, utiliza-se um conjunto de equações dinâmicas que captam o efeito passado das variáveis, por meio da metodologia de equações aparentemente não correlacionadas.

\section{ara atingir as características finais do produto, uma equação de realimentação foi estabelecida}

Um outro aspecto importante é a ligação entre o controle estatístico e o controle de engenharia do processo, pois, em primeiro lugar, investiga-se sobre a estabilidade do processo e, depois, procura-se fazer a previsão da variável de interesse, para se tomar uma ação antecipada, evitando-se, assim, o desperdício.

Para a realização deste trabalho, inicialmente, a estabilidade do processo produtivo foi avaliada por um gráfico multivariado $\mathrm{T}^{2}$ de Hotelling e, se o processo fosse considerado instável, uma busca pela variável, ou pelo conjunto de variáveis responsáveis por essa instabilidade, por meio da análise de componentes principais e do gráfico X-barra.

Após a identificação das variáveis causadoras de instabilidade no processo, e verificando-se que as causas que estavam fazendo com que o mesmo produzisse itens com defeitos não eram passíveis de remoção, passou-se ao emprego do controlador, conforme detalhado no item a seguir. Mas, antes de sua aplicação, foram estimadas as equações de regressões aparentemente não-correlacionadas, pois os erros das equações mostraram-se correlacionados, e essa metodologia proporcionou a ponderação dos mesmos. Os distúrbios do processo foram previstos utilizando-se a estatística de médias móveis exponencialmente ponderadas, pois revelam o comportamento dos distúrbios, e a constante de impacto dos sistema foi determinada por meio da equação de regressão, que representava a variável em estudo.

A metodologia proposta foi empregada em uma empresa do setor cerâmico, que produz azulejo de diversos tipos. Os dados foram coletados em um forno de queima, 
do tipo túnel, com 85 metros de comprimento, onde foi possível observar doze variáveis de temperatura, cada uma composta de 92 observações. Os valores nominais dessas temperaturas são preestabelecidos, para que ocorra a queima de materiais cerâmicos. Após a coleta dos dados, as variáveis em estudo foram submetidas a uma avaliação da sua estabilidade por meio de um gráfico $\mathrm{T}^{2}$ de Hotelling. Se o processo fosse considerado estável, passaria para a etapa da monitoração da produção. Caso contrário, tentar-se-ia identificar as possíveis causas, ou as possíveis variáveis, que estariam afetando o processo.

A etapa seguinte é conhecer o comportamento futuro da variável causadora de instabilidade, que é realizada por meio da análise de regressão aparentemente não correlacionada. Logo que seus valores futuros são conhecidos, aplica-se o controlador proposto, efetuando-se, assim, a realimentação nas variáveis identificadas, mantendo-se o processo o mais próximo do alvo estipulado.

\section{AJUSTE DE REALIMENTAC̄̃̃O MULTIVARIADA}

O Controle Estatístico de Processos (CEP) tenta identificar, e remover, as causas especiais que fazem com que o processo esteja fora de controle. O Controle de Engenharia do Processo (CEnP), amplamente discutido por Box (1991), Box et al. (1978), Box et al. (1994), baseiase em métodos de estimação das variáveis envolvidas no processo, pois o conhecimento do comportamento futuro das variáveis permite realizar os ajustes necessários no sistema, mantendo a sua estabilidade, sendo que estes autores dedicam-se ao caso univariado e, aqui, faz-se uma extensão para o caso multivariado, onde um conjunto de variáveis é utilizado para se realizar o ajuste de realimentação.

Os processos de ajuste, que utilizam o distúrbio produzido para correção do sistema, são denominados de controle de realimentação, pois os desvios do alvo encontrados, no processo, são utilizados para realimentar o sistema. A maioria dos trabalhos, envolvendo controle de processos, nas indústrias, é feita utilizando controladores proporcionais, ou integrais ao erro e, segundo Shinskey (1994), Ramirez (1994) e Del Castillo (1996), isso continuará sendo verdade no futuro, devido a sua simplicidade e robustez. Neste trabalho, utiliza-se um controlador proporcional ao erro, conforme é mostrado a seguir.

A realimentação do sistema proporciona que as medidas corretivas sejam quantificadas, para, posteriormente, serem efetivadas. Essas medidas podem ser realizadas obedecendo à expressão (1), que é denominada de controlador PI (proporcional - integral), pois a ação de controle pode ser atingida se aplicada uma ação utilizando a parcela proporcional, ou a parcela integral.

$$
g X_{t}=k_{0}+k_{P} e_{t}+k_{I} \sum_{i=1}^{t} e_{i}
$$

Onde:

- $X_{t}$ representa a variável que sofrerá o ajuste;

- g representa o efeito que as alterações de uma unidade efetuadas na variável de entrada causarão no sistema, sendo denominado de ganho do sistema;

- $k_{P} \mathrm{e} k_{I}$ são constantes que correspondem à proporção com que cada termo do controlador contribuirá para a ponderação da combinação linear (BOX, 1991; BOX; LUCEÑO, 1997);

- $e_{t}$ e e $e_{i}$ são os erros de previsão dos distúrbios, ou desvios $d_{t}$ encontrados; $\mathrm{e}$

- $d_{t}=X_{t}-A$ são os desvios do alvo de uma variável, em que $X_{t}$ representa a variável que está sendo analisada, e $A$ é o valor alvo para essa variável.

No controle de realimentação, nos períodos anteriores ao tempo $t$, que é o tempo em que a ação ocorrerá, têm-se os erros dos distúrbios previstos, representados por ...e $e_{t}$ $e_{t-1}, e_{t-2} \ldots$, os quais determinam o nível em que a variável de entrada $\boldsymbol{X}_{t}$ deve ser manipulada, para que os mesmos sejam os menores possíveis. Na realidade, o que deve ser feito, sempre que possível, é cancelar o distúrbio $\boldsymbol{d}_{t}$, através do ajuste da variável $\boldsymbol{X}_{\boldsymbol{t}}$, de modo que o processo se mantenha próximo do alvo desejado. Logo, escreve-se (2)

$$
X_{t+1}-A=d_{t+1}+g X_{t}
$$

Essa relação mostra que, no instante $t$, o desvio do alvo $\mathbf{X}_{t+1}-\mathbf{A}$ depende do distúrbio $\mathbf{d}_{t+1}$, e do nível de ajuste $\mathbf{g X}_{\mathrm{t}}$, que a variável $\mathrm{X}_{\mathrm{t}}$ sofreu no instante $t$.

Observando-se a relação (2), no instante $t$, tem-se que se deve ajustar a variável $\boldsymbol{X}_{\boldsymbol{t}}$, de modo que o lado direito da relação torne-se zero. Sendo assim, não haverá desvios do alvo no instante $t+l$ e $\mathbf{X}_{\mathrm{t}+1}-\mathbf{A}$, será igual a zero. Infelizmente isso não pode ser efetivado, pois no instante $t$ não se conhece o valor de $\mathbf{d}_{\mathbf{t}+1}$. Entretanto, no instante $t$ pode ser feita a previsão do distúrbio, $\hat{\mathbf{d}}_{\mathrm{t}+1}$, e pode-se escrever que $\mathbf{e}_{\mathbf{t}+1}=\mathbf{d}_{\mathbf{t}+\mathbf{1}}-\hat{\mathbf{d}}_{\mathbf{t}+1}$ é o erro de previsão do distúrbio. Logo, a relação (2) pode ser escrita como em (3):

$$
\mathbf{X}_{t+1}-\mathbf{A}=\mathbf{e}_{t+1}+\hat{\mathbf{d}}_{\mathrm{t}+\mathbf{1}}+\mathbf{g X _ { t }}
$$

Na realidade, o que necessita ser feito é ajustar $X_{t}$, de modo que 


$$
\mathbf{g X}_{\mathrm{t}}=-\hat{\mathbf{d}}_{\mathbf{t}+\mathbf{1}}
$$

substituindo-se (4) em (3), tem-se

$$
\mathbf{X}_{\mathbf{t}+\mathbf{1}}-\mathbf{A}=\mathbf{e}_{\mathbf{t}+\mathbf{1}}
$$

Isso mostra que o desvio do alvo, visto no processo, será o erro de previsão do distúrbio. A relação (4) mostra o ajuste feito no instante $t$, mas se o ajuste tiver que ser realizado em um instante anterior tem-se que:

$$
g\left(X_{t}-X_{t-1}\right)=-\left(\hat{d}_{t+1}-\hat{d}_{t}\right)
$$

mas sabe-se que $\left(\hat{\mathbf{d}}_{\mathbf{t}+\mathbf{1}}-\hat{\mathbf{d}}_{\mathbf{t}}\right)$ não significa o valor do distúrbio e, sim, o erro que se comete em prever o distúrbio. Essa diferença pode ser modelada segundo a estatística de médias móveis exponencialmente ponderada, que em inglês denomina-se Exponentially Weighted Moving Average (EWMA). Assumindo a forma mostrada em (7):

$$
\left(\hat{d}_{t+1}-\hat{d}_{t}\right)=\lambda\left(d_{t}-\hat{d}_{t}\right)=\lambda e_{t}
$$

onde $\lambda$ representa a constante de ponderação da estatística de médias móveis, e o valor de $\lambda$ utilizado será aquele que fornecer o menor erro de previsão da série dos erros dos distúrbios ajustados, fornecidos por esta estatística. Fazendo-se a substituição de (7) em (6), obtém-se a expressão de ajuste do sistema, representada pela expressão:

$$
g\left(X_{t}-X_{t-1}\right)=-\lambda e_{t}
$$

onde $\boldsymbol{e}_{t}$ representa o erro de previsão dos distúrbios. Desenvolvendo-se um pouco mais a expressão (8), encontra-se:

$$
X_{t}-X_{t-1}=-\frac{\lambda}{g}\left(d_{t}-\hat{d}_{t}\right)
$$

Fazendo-se a comparação da expressão de realimentação em (1), e escrevendo-se a expressão (8) como $\mathbf{X}_{\mathbf{t}}=\mathbf{X}_{\mathbf{t}-\mathbf{1}}+\frac{\lambda}{\mathbf{g}} \mathbf{e}_{\mathbf{t}}$ pode-se dizer que se estabeleceu um controle proporcional ao erro, no caso discreto. Essa proporcionalidade é a quantidade que mede a diferença entre o valor que a variável deveria apresentar e o valor corrente da mesma.

A idéia de se trabalhar com o ajuste proporcional ao último erro deve-se ao fato de que ele é a previsão do distúrbio, e esse ajuste ocorrerá a cada etapa, para cancelar esta previsão. Utilizando-se a constante de ponderação $\lambda$ aplicada à observação mais recente, ou seja, ao último erro, evita que haja uma compensação excessiva, no sistema (BOX; LUCEÑO, 1994, 1997 e MONTGOMERY et. al. 1994). Como os distúrbios são previstos, e a cada etapa eles são cancelados, logo não há a necessidade de se fazer um controle integral, que representaria a soma de todos os resíduos passados, os quais não influenciarão no sistema, pois além de já estarem corrigidos, não conheceríamos os reais distúrbios que afetariam o sistema.

Segundo Box \& Luceño (1997), ao invés de se considerar o nível de entrada da variável $\boldsymbol{X}_{t}$ no tempo $t$, podese pensar em termos de ajustamentos onde $\mathbf{x}_{\mathbf{t}}=\mathbf{X}_{\mathbf{t}}-\mathbf{X}_{\mathrm{t}-1}$ a ser feito no tempo $t$, reescrevendo-se (8) como:

$$
\mathbf{g x}_{\mathbf{t}}=-\lambda \mathbf{e}_{\mathbf{t}}
$$

onde verifica-se que um ajuste proporcional, em relação ao último erro, foi realizado.

$\mathrm{O}$ ajuste proporcional pode ser verificado, considerando a expressão (1) com os índices de tempos $t$ e $t$ - 1 . Ao realizar a subtração, considerando-se o instante atual e o anterior, obtém-se:

$$
g\left(X_{t}-X_{t-1}\right)=\left(k_{p}+k_{I}\right) e_{t}-k_{p} e_{t-1}
$$

podendo ser escrito como:

$$
g x_{t}=c_{1} e_{t}+c_{2} e_{t-1}
$$

onde $\mathbf{c}_{\mathbf{1}}=\mathbf{k}_{\mathbf{p}}+\mathbf{k}_{\mathbf{1}}$ e $\mathbf{c}_{\mathbf{2}}=-\mathbf{k}_{\mathrm{p}}$ consiste em fazer um ajustamento na variável de entrada, a qual é uma combinação dos dois últimos erros.

Comparando-se a expressão (12) com (10), tem-se que $\mathbf{g} \mathbf{x}_{\mathbf{t}}=-\boldsymbol{\lambda} \mathbf{e}_{\mathbf{t}}$ segue um ajuste proporcional ao erro, onde $\mathbf{c}_{\mathbf{1}}=-\boldsymbol{\lambda}$ e $\mathbf{c}_{\mathbf{2}}=\mathbf{0}$ (BOX \& LUCEÑO, 1997).

Como os dados amostrais são coletados e medidos em tempos equiespaçados, e os ajustes também serão feitos em tempos equiespaçados, em relação ao distúrbio de cada variável, considera-se, então, um sistema de ajuste discreto em relação ao distúrbio de cada variável. Dessa forma, a equação (9) fornece o nível de ajuste que deve ser feito na variável de compensação. A constante g representa o ganho do sistema, representada pelo maior coeficiente da equação de regressão, que explica aquela variável. A constante é estimada através do modelo de equações aparentemente não correlacionadas, denominadas, em inglês, por Vector Autoregression $(V A R)$. Dessa forma, capta-se o efeito conjunto das variáveis.

\section{Realização e interpretação do controlador proposto}

O sucesso de um ajuste requer dois esforços: um processo de identificação e uma aplicação apropriada das 
regras de ajustes. A identificação das variáveis será feita com a utilização do gráfico $\mathrm{T}^{2}$ de Hotelling e da Análise de Componentes Principais $(A C P)$. Pois, quando se verificou que o processo foi considerado instável, pelo gráfico $\mathrm{T}^{2}$, passou-se à etapa de investigação das possíveis variáveis causadoras dessa instabilidade. Realizou-se a $\mathrm{ACP}$, e essas foram avaliadas por meio de um gráfico Xbarra, que foi capaz de mostrar quais estavam fora de controle. As componentes consideradas fora de controle, foram submetidas a um estudo de correlação entre as suas variáveis originais e suas componentes instáveis, revelando, assim, as variáveis que seriam as prováveis candidatas de estarem causando estabilidade no sistema.

Como se deseja captar as inter-relações entre as variáveis, utiliza-se a variável anterior àquela identificada como fora de controle e a variável posterior. No caso em que se está trabalhando com variáveis que se apresentam distribuídas seqüencialmente, então, um conjunto de três variáveis será utilizado. Sendo estas consideradas dependentes, as outras variáveis do sistema entrarão como variáveis independentes, utilizando-se a metodologia de estimação do geral para o específico, isto é, em cada equação utilizaram-se as variáveis inicialmente com quatro defasagens, as quais foram eliminadas considerandose a sua significância estatística no modelo. As estimadas por meio da metodologia Quasi VAR trazem um ganho em eficiência nas estimativas (ZELLNER; THEIL, 1962).

nando, assim, uma maior liberação de energia no processo. Se o sinal for negativo (-) significa que uma redução deve ser introduzida no sistema. Logo, o botão regulador deverá ser girado no sentido anti-horário.

O valor numérico, resultante da expressão (9), indicará o número de unidades que o botão de controle deve ser girado, pois o mesmo é dotado de uma escala de medida que facilita a regulagem.

No sistema multivariado, todas as variáveis selecionadas deverão sofrer o ajuste de realimentação, devendo-se salientar que cada uma delas terá o seu próprio valor de ajuste.

Dessa forma, procura-se estabelecer uma rotina criteriosa de calibração das variáveis de saída, com o objetivo de mantê-las o mais próximo possível do alvo estipulado.

\section{MODELOS AUTO-REGRESSIVOS MULTIVARIADOS}

Pesquisadores e trabalhadores do campo industrial, freqüentemente obtêm dados que apresentam diversas respostas para um determinado processo, sendo que esse conjunto de variáveis deve ser controlado. Quando a estrutura da resposta é multivariada, surge um problema na estimação dos parâmetros não existente no caso univariado, pois o vetor de parâmetros, a ser estimado, deve levar em consideração as inter-relações entre as variáveis (KHURI; CONLON, 1981). A metodologia de regressões, aparentemente não correlacionadas, possibilita uma estimação conjunta dos parâmetros, onde as inter-relações são consideradas

Depois de identificadas as variáveis, que serão ajustadas por meio do controlador, devem-se encontrar os valores-alvos para cada lote de fabricação. Esse valor será representado, genericamente, pela letra $A$, e o objetivo será manter o processo o mais próximo possível deste valor $A$, que será efetivado pela manipulação das variáveis de entrada.

O nível de controle, a ser introduzido no processo, será dado pela expressão (9), que mostra a relação entre o nível das variáveis de entrada e o distúrbio produzido na saída do processo. Considerando que a maioria dos processos de controle são feitos por meio da regulagem de um botão, no painel de controle, aqui, mostra-se como esta metodologia proposta pode ser efetivada.

$\mathrm{Na}$ expressão (9), se o resultado numérico apresentar sinal positivo (+) indicará que o botão regulador do sistema deverá ser girado no sentido horário, proporcio- e o comportamento dinâmico dos dados é capturado, fornecendo um conhecimento da estrutura de relação entre as variáveis de entrada e de saída do sistema.

Essa metodologia, também denominada de Vetores Auto-regressivos $(V A R)$, possibilita a análise não somente do comportamento individual de cada série, mas também das possíveis relações existentes entre as séries e as relações dinâmicas que ocorrem entre elas, em um determinado período de tempo. Dessa maneira, torna-se possível aumentar a acurácia das estimativas do modelo, utilizando-se as informações adicionais fornecidas pelas inter-relações, fornecendo uma medida confiável, para que o ajuste de realimentação seja realizado. Os modelos, tanto univariados como multivariados, são bastante discutidos por autores como Box; Jenkins (1970), Lütkepohl (1991), Maddala (1992), Charemza \& Deadman (1997) E Reinsel (1993), Hamilton (1994), que 
mostram o caso multivariado como uma generalização do univariado.

Um vetor auto-regressivo é, simplesmente, um sistema de equações lineares dinâmicas, em que cada variável é escrita como função de um erro serialmente nãocorrelacionado, e todas as variáveis, que pertencem ao sistema, possuem o mesmo número de defasagens, representadas por $p$. Essas defasagens determinam a ordem do modelo, que, genericamente, é representado por $\operatorname{VAR}(p)$, tal como pode ser visto em (13).

$$
Z_{t}=v+\varphi_{1} Z_{t-1}+\ldots+\varphi_{p} Z_{t-p}+\varepsilon_{t}
$$

Na equação (13) tem-se que $\boldsymbol{Z}_{t}$ é um vetor aleatório, $\varphi_{i}$ é a matriz dos coeficientes, $v$ é o vetor dos interceptos, o qual permite que a média do processo seja diferente de zero e $\varepsilon_{t}$ é o vetor ruído branco, também chamado vetor das inovações do processo, isto é: $\boldsymbol{E}\left(\varepsilon_{t}\right)=\boldsymbol{0}$ e $\boldsymbol{E}\left(\boldsymbol{\varepsilon}_{t} \boldsymbol{\varepsilon}_{t}{ }_{t}\right)=\boldsymbol{\Sigma}$, onde $\boldsymbol{\Sigma}$ é a matriz de variância-covariância não-singular $E\left(\varepsilon_{t} \varepsilon^{\prime}\right)=0$ para $s \neq t$.

Para explicitarmos como o vetor auto-regressivo será estimado, e como os seus erros se comportam, será utilizado um modelo auto-regressivo de primeira ordem, $\operatorname{VAR}(1)$, descrito a seguir:

$$
Z_{t}=v+\varphi_{1} Z_{t-1}+\varepsilon_{t}
$$

tomando-se o fator tempo, $t=1,2, \ldots, t$, podem-se escrever as seguintes equações:

$Z_{1}=v+\varphi_{1} Z_{0}+\varepsilon_{1}$
$Z_{2}=v+\varphi_{1} Z_{1}+\varepsilon_{2}$

\begin{tabular}{|c|c|}
\hline $\begin{array}{l}Z_{2}=v+\varphi_{1}\left(v+\varphi_{1} Z_{0}+\varepsilon_{1}\right)+\varepsilon_{2} \\
=\left(I_{k}+\varphi_{1}\right) v+\varphi^{2} Z_{1}+\varphi_{1} \varepsilon_{1}+\varepsilon_{2}\end{array}$ & (17) \\
\hline$\vdots$ & \\
\hline$Z_{t}=\left(I_{k}+\varphi_{1}+\ldots+\varphi_{1}^{t-1}\right) \cup+\varphi_{1}^{t} Z_{0}+\sum_{i=0}^{t-1} \varphi_{1}^{i} \varepsilon_{t-i}$ & (18) \\
\hline
\end{tabular}

substituindo-se (14) em (15), tem-se a equação:

Observando-se as equações (17) e (18), vê-se que o processo auto-regressivo é determinado por um valor inicial, seguido dos choques aleatórios anteriores. Os vetores $\boldsymbol{Z}_{1}, \ldots, \boldsymbol{Z}_{t}$ são determinados, unicamente, por $\mathbf{Z}_{0}$, que é o valor inicial mais a soma dos $\varepsilon s$.

Assim, vê-se que o modelo multivariado (18) poderá ser representado por uma soma infinita de erros defasados, mais o valor de $\varphi_{1}{ }^{t} Z_{0}$, que tenderá a zero quando $t$ tender ao infinito. Dessa forma, o vetor auto-regressivo infinito poderá ser melhor denominado de vetor médias móveis finito. O modelo $\operatorname{VAR}(1)$, que apresenta os seus autovalores $\varphi_{1}$ menores do que 1 , terá os seus parâmetros estáveis, o que é uma condição satisfatória para o modelo fornecer boas previsões (COCHRANE, 1997).

O sistema multivariado deve apresentar um processo ruído branco, de forma que os erros sejam independentes e identicamente distribuídos, ou seja, $\boldsymbol{\varepsilon}_{t} \approx \boldsymbol{i i d} \mathbf{N}(\boldsymbol{0}, \Sigma)$. Essa condição garante a ausência de qualquer correlação serial nos erros, quer dizer, os resíduos sejam homoscedásticos, apresentando uma variância constante.

Considerando-se o exemplo de um sistema composto por duas variáveis (x e y) com uma defasagem, torna-se, assim, mais compreensível o processo $\operatorname{VAR}(1)$, descrito a seguir:

$$
\left[\begin{array}{l}
x_{t} \\
y_{t}
\end{array}\right]=\left[\begin{array}{ll}
a_{1} & b_{1} \\
c_{1} & d_{1}
\end{array}\right]\left[\begin{array}{l}
x_{t-1} \\
y_{t-1}
\end{array}\right]+\left[\begin{array}{l}
\varepsilon_{1 t} \\
\varepsilon_{2 t}
\end{array}\right]
$$

Essa forma matricial pode ser reescrita da seguinte forma:

$$
\begin{aligned}
& x_{t}=a_{1} x_{t-1}+b_{1} y_{t-1}+\varepsilon_{1 t} \\
& y_{t}=c_{1} x_{t-1}+d_{1} y_{t-1}+\varepsilon_{2 t}
\end{aligned}
$$

Observa-se que ambas as variáveis defasadas, $x$ e $y$, aparecem, simultaneamente, em cada equação. Logo o vetor $A R(1)$ capta o efeito dinâmico nas inter-relações das variáveis. $\mathrm{O}$ vetor auto-regressivo de primeira ordem, representado pela equação (13), também pode ser visualizado em termos de notação vetorial, como mostra a equação (21).

$$
\mathbf{Z}_{\mathrm{t}}=\varphi \mathbf{Z}_{\mathrm{t}-\mathbf{1}}+\boldsymbol{\varepsilon}_{\mathrm{t}}
$$

Observando-se o modelo multivariado (20), nota-se que os erros das equações estão correlacionados durante o mesmo período de tempo, pois as mesmas variáveis pertencem às duas equações. Isso pode levar a conclusões errôneas no momento de se tomar uma decisão. Para que isso seja evitado, a melhor maneira de se neutralizar essas correlações seria tornar os erros ortogonais, tornando as equações independentes. A ortogonalização possibilita a correta interpretação das previsões fornecidas pelo modelo $V A R$, pois as correlações existentes entre os resíduos e o efeito das outras variáveis serão neutralizadas. Dessa forma, cada equação pode ser utilizada separadamente, sem que exista o efeito da correlação.

Segundo Charenza; Deadman (1997), pode-se observar que os termos dos erros são contemporaneamente 
correlacionados, isto é, $E\left(\varepsilon_{1 t}\right)=E\left(\varepsilon_{2 t}\right)=0 ; \quad E\left(\varepsilon_{1 t}^{2}\right)=\sigma_{11}$; $E\left(\varepsilon_{2 t}^{2}\right)=\sigma_{22} ; E\left(\varepsilon_{1 t} \varepsilon_{2 t}\right)=\sigma_{12}$. A fim de obter-se os erros descorrelacionados, uma ponderação deve ser feita por meio da multiplicação da primeira linha do sistema (20) pelo fator $\delta=\frac{\sigma_{12}}{\sigma_{11}}$, que logo após é subtraído do resultado da segunda linha do sistema, obtendo-se:

$$
\left[\begin{array}{c}
x_{t} \\
y_{t}-\delta x_{t}
\end{array}\right]=\left[\begin{array}{cc}
a_{1} & b_{1} \\
c_{1}^{*} & d_{1}^{*}
\end{array}\right]\left[\begin{array}{c}
x_{t-1} \\
y_{t-1}
\end{array}\right]+\left[\begin{array}{c}
\varepsilon_{1 t} \\
\varepsilon_{2 t}^{*}
\end{array}\right]
$$

onde: $c^{*}{ }_{i}=\left(c_{i}-\delta a_{i}\right) ; d^{*}{ }_{i}=\left(d_{i}-\delta b_{i}\right) ; \varepsilon^{*}{ }_{2 t}=\left(\varepsilon_{2 t}-\delta \varepsilon_{2 t}\right) ; \mathrm{com}$ $i=1$ para este exemplo específico; mas o mesmo pode ser expandido para $i=1,2, \ldots$.

Em (22), os valores $\varepsilon_{1 t}$ e $\varepsilon_{2 t}^{*}$ são não-correlacionados, pois $\left.E\left(\varepsilon_{1 t} \varepsilon^{*}{ }_{2 t}\right)=\left(\varepsilon_{1 t}\left(\varepsilon_{2 t}-\delta \varepsilon_{1 t}\right)\right)=E\left(\varepsilon_{1 t} \varepsilon_{2 t}^{*}\right)-\left(\sigma_{12} / \sigma_{11}\right) E\left(\varepsilon^{2}{ }_{1 t}\right)\right)$ $=\sigma_{12}-\sigma_{12}=0$.

Essa apresentação deixa claro que a inter-relação entre os erros é neutralizada pela ponderação das variáveis através das variâncias e covariâncias dos erros.

Segundo Enders (1995), se algumas das equações possuírem regressores não incluídos nos outros, variáveis diferentes do lado direito de cada equação, ou até mesmo se as variáveis possuírem defasagens diferentes, deve-se utilizar o estimador de regressões aparentemente não correlacionadas, que vem do inglês como Seegmingly Unrelated Regression (SUR), se desejarmos obter uma boa eficiência nas estimativas dos coeficientes VAR, obtendo-se um modelo denominado de Quasi VAR, que vem do termo original em inglês "near VAR". A forma, de como estes parâmetros são estimados será mostrada a seguir.

\section{Previsão com os modelos auto-regressivos multivariados}

Uma aplicação direta dos modelos auto-regressivos, diz respeito à previsão de séries temporais multivariadas. Quando se utiliza as previsões um passo-à-frente, os valores utilizados até o período $t$ são reutilizados para realizar previsões para o período $t+1$.

Seja $Z_{t+1 / t}^{*}$ a previsão de $Z_{t+1}$, baseado em $X_{t}$, tem-se que avaliar a utilidade dessa previsão, baseando-se em uma função perda. Os resultados mais convenientes, e amplamente utilizados, são aqueles que assumem uma função perda quadrática, indicando que o valor escolhido da previsão $Z_{t+1 / t}^{*}$ é o mínimo, conforme mostra (24), que é conhecida como Erro Quadrático Médio (EQM), associado com a previsão $Z_{t+1 / t}^{*}$.

$$
\operatorname{EQM}\left(Z_{t+1 / t}^{*}\right)=E\left(Z_{t+1}-Z_{t+1 / t}^{*}\right)^{2}
$$

A previsão com o menor $E Q M$ será o valor esperado de $Z_{t+1}$, condicionado a $X_{t}$, que apresentará os melhores valores previstos.

Considerando-se um conjunto de dados que segue um modelo $\operatorname{VAR}(1)$, e que são conhecidos todos os valores passados das variáveis, será possível conhecer seus valores futuros, procurando-se, sempre, o menor valor para o $E Q M$, garantindo, assim, os melhores valores previstos para as variáveis.

Considera-se o processo $\operatorname{VAR}(1)$, representado na equação (21), sabendo-se que o mesmo pode ser generalizado por meio de sucessivas substituições e apresentado como em (18). Assim, tem-se:

$$
Z_{t+h}=\varphi_{1}^{h} Z_{t}+\sum_{i=0}^{h-1} \varphi_{1}^{i} \varepsilon_{t+h-i}
$$

onde $h$ representa o horizonte de previsão desejado.

A partir do preditor $Z_{t}(h)=B_{0} Z_{t}+B_{1} Z_{t-1}+\ldots$, onde $B_{i}$ pode ser visto como a matriz dos coeficientes, o erro de previsão será dado por:

$$
Z_{t+h}-Z_{t}(h)=\sum_{i=0}^{h-1} \varphi_{1}^{i} \varepsilon_{t+h-i}+\left(\varphi_{1}^{h}-B_{0}\right) Z_{t}-\sum_{i=1}^{\infty} B_{i} Z_{t-i}
$$

Sabendo-se que $\mathrm{e}_{t+j}$ para $j>0$ é não-correlacionado de $Z_{t-1}$, para $i \geq 0$, tem-se:

$$
\begin{aligned}
& \operatorname{EQM}\left(Z_{t}(h)\right)=E\left(\sum_{i=0}^{h-1} \varphi_{1}^{i} \varepsilon_{t+h-i}\right)\left(\sum_{i=0}^{h-1} \varphi_{1}^{i} \varepsilon_{t+h-i}\right)^{\prime} \\
& E\left[\left(\varphi_{1}^{h}-B_{0}\right) Z_{t}-\sum_{i=1}^{\infty} B_{i} Z_{t-i}\right]\left[\left(\varphi_{1}^{h}-B_{0}\right) Z_{t}-\sum_{i=1}^{\infty} B_{i} Z_{t-i}\right]^{\prime}
\end{aligned}
$$

A matriz com menor EQM é construída quando $B_{0}=\varphi_{1}{ }^{h}$ e $B_{i}=0$ para i $>0$. Então, o preditor ótimo será obtido por:

$$
Z_{t}(h)=\varphi_{1}^{h} Z_{t}=\varphi_{1} Z_{t}(h-1)
$$

Para que seja possível avaliar o efeito que uma variável pode causar em uma outra, deve-se tornar a média do sistema igual a zero, onde é considerado $v=0$, na equação (14), obtendo-se a equação (21). Supondo-se um sistema composto de três variáveis $Z_{1}, Z_{2}$ e $Z_{3}$, para isolar o efeito que cada uma possa causar à outra, e supondo-se que $Z_{1}$ aumente de uma unidade no instante de tempo de $t=0$; isto é, $\varepsilon_{1,0}=1$, é possível observar o que acontece no sistema durante o período de tempo $t=1,2, \ldots$. Se nenhum choque, ou inovação, ocorrer no sistema, significa dizer que $\varepsilon_{2,0}=\varepsilon_{3,0}=0, \varepsilon_{1}=0, \varepsilon_{2}=0$, desde que não se está interessado na média do sistema, mas nas inovações das variáveis em torno das médias. 
Continuando o procedimento para $Z_{i}=\left(Z_{1, i} ; Z_{2, i} ; Z_{3, i}\right)^{\prime}$, tem-se, na primeira coluna, o vetor dos parâmetros $\varphi_{1}^{i}$, que representam o impacto causado no sistema. Uma linha de argumento mostra que o choque em $Z_{2 i}$, no instante $\mathrm{t}=0$, após os $i$ 's períodos, resulta na segunda coluna de $\varphi_{1}^{i}$, e assim sucessivamente. Por esse motivo, eles são chamados de respostas de impulsos, ou multiplicadores dinâmicos. Em outras palavras, $\varphi$ representa a reação da $n$-ésima variável do sistema, quando ela recebe um choque, ou uma inovação, num período anterior.

Sempre que houver uma inovação ortogonal, a reação que uma variável receberá também será do tipo ortogonal, e cada equação poderá ser tratada independentemente uma da outra.

\section{Estimação dos parâmetros de regressão aparentemente não-correlacionadas}

Como pode ser observado, na estimação das equações multivariadas, o sistema apresenta uma estrutura fixa, com as mesmas variáveis explanatórias em todas as equações e com o mesmo número de defasagens. Por esse motivo, a metodologia proposta por Sims (1980) recebeu muitas críticas, por não ser possível, na prática, evitar-se a imposição de certas restrições ao sistema VAR. Essas restrições, na maioria das vezes, estão relacionadas ao número de variáveis que devem ser incluídas no modelo e ao número de defasagens que deve ser aceito em cada uma (KEATING, 1990).

Sobre as condições de simultaneidade, descritas anteriormente, Zellner (1962) demonstra que o método de regressões aparentemente não-correlacionadas (SUR) possibilita fazer uma estimação assintoticamente mais eficiente do que se fosse estimar equação por equação.

Uma suposição que permite utilizar um processo de estimação conjunta - que é melhor do que a estimação de mínimos quadrados separada - é a ligação das equações através dos erros. Essa suposição afirma que os termos estocásticos nas equações, no mesmo instante, são correlacionados. O fato de se acrescentar a suposição de correlação contemporânea produz o efeito de introduzir informação adicional não incluída quando se faz separadamente a estimação de mínimos quadrados (HILL et al., 1999). A utilização do $S U R$ lança mão da informação da correlação entre os termos estocásticos, por isso ela é mais precisa do que o processo de mínimos quadrados, e esse fato é corroborado pelos desvios-padrões menores das estimativas.

O método de estimação $S U R$ possibilita que cada equação tenha a sua própria forma funcional, levando em consideração apenas a correlação existente entre os erros das equações. Descreve-se, aqui, o método $S U R$ de estimação, como originalmente feito por Zellner (1962), levando-se em consideração o sistema multivariado representado em (27).

$$
\left[\begin{array}{c}
Y_{1} \\
Y_{2} \\
\vdots \\
Y_{M}
\end{array}\right]=\left[\begin{array}{cccc}
X_{1} & 0 & \cdots & 0 \\
0 & X_{2} & \cdots & 0 \\
\vdots & \vdots & \ddots & \vdots \\
0 & 0 & \cdots & X_{M}
\end{array}\right]\left[\begin{array}{c}
\beta_{1} \\
\beta_{2} \\
\vdots \\
\beta_{M}
\end{array}\right]+\left[\begin{array}{c}
u_{1} \\
u_{2} \\
\vdots \\
u_{M}
\end{array}\right]
$$

onde $Y_{u}$ representa o vetor das observações dependentes; $X_{M}$ representa o vetor das observações independentes, $\beta_{M}$ é o vetor dos coeficientes e $U_{M}$ é o vetor dos termos dos erros aleatórios, cada um com média zero. $\mathrm{O}$ sistema (27) pode ser reescrito na forma vetorial conforme (28),

$$
\mathbf{Y}=\mathbf{X} \boldsymbol{\beta}+\mathbf{u}
$$

onde $\quad Y \equiv\left[\begin{array}{llll}Y_{1}^{\prime} & Y_{2}^{\prime} & \cdots & Y_{M}^{\prime}\end{array}\right] ; \quad \beta \equiv\left[\begin{array}{llll}\beta_{1}^{\prime} & \beta_{2}^{\prime} & \cdots & \beta_{M}^{\prime}\end{array}\right]$; $u \equiv\left[\begin{array}{llll}u_{1}^{\prime} & u_{2}^{\prime} & \cdots & u_{M}^{\prime}\end{array}\right]$ e $X$ representa a matriz blocodiagonal de (27). O vetor de distúrbio (MT x 1) em (27) e (28) possui a seguinte matriz de covariância:

$$
\begin{gathered}
\Sigma=V(u)=\left[\begin{array}{cccc}
\sigma_{11} I & \sigma_{12} I & \cdots & \sigma_{1 M} I \\
\sigma_{21} I & \sigma_{22} I & \cdots & \sigma_{2 M} I \\
\vdots & \vdots & & \vdots \\
\sigma_{M 1} I & \sigma_{M 2} I & \cdots & \sigma_{M M} I
\end{array}\right]=\left[\begin{array}{cccc}
\sigma_{11} & \sigma_{12} & \cdots & \sigma_{1 M} \\
\sigma_{21} & \sigma_{22} & \cdots & \sigma_{2 M} \\
\vdots & \vdots & & \vdots \\
\sigma_{M 1} & \sigma_{M 2} & \cdots & \sigma_{M M}
\end{array}\right] \otimes I \\
=\Sigma_{c} \otimes I
\end{gathered}
$$

onde $I$ é a matriz identidade de ordem $(M \times M)$ e $\sigma_{u u^{\prime}}=\mathrm{E}$ $\left(u_{u t} u_{u^{\prime} t}\right)$ para $t=1,2, \ldots, T$ e $u, u^{\prime}=1,2, \ldots, M$. No modelo (28), supõe-se que as variâncias são constantes de um período para outro, havendo a ausência de auto-correlação residual. Os valores $\sigma_{u u^{\prime}}$ para $u=u^{\prime}$ representam as variâncias, sendo que para $u \neq u^{\prime}$ representam as covariâncias do distúrbio das variáveis dependentes para qualquer período.

Nas equações (27) e (28) é aplicado o Método dos Mínimos Quadrados Generalizados (MMQG), pois dessa forma é feita a estimação simultânea dos parâmetros das equações. Para que isso seja possível, ambos os lados da equação (27) são multiplicados por uma matriz $H$, de tal forma que $\mathrm{E}\left(\mathrm{Huu} \mathrm{H}^{\prime}\right)=\mathrm{H} \Sigma \mathrm{H}^{\prime}=\mathrm{I}$. Em termos das variáveis transformadas, ou seja, as variáveis originais prémultiplicadas por $H$, o sistema satisfaz as suposições do Modelo de Mínimos Quadrados Ordinários. Esta aplicação irá conduzir a um estimador não viesado, dado por (30). 


$$
b^{*}=\left(X^{\prime} H^{\prime} H X\right)^{-1} X^{\prime} H^{\prime} H Y=\left(X^{\prime} \Sigma^{-1} X\right)^{-1} X \Sigma^{-1} Y
$$

na construção desse estimador, necessita-se da inversa de $\Sigma$, a qual é dada por

$$
\Sigma^{-1}=V^{-1}(u)=\left[\begin{array}{ccc}
\sigma^{11} I & \cdots & \sigma^{1 M} I \\
\vdots & & \vdots \\
\sigma^{M 1} I & \cdots & \sigma^{M M} I
\end{array}\right]=\Sigma_{c}^{-1} \otimes I
$$

Então, o estimador do vetor dos coeficientes, dado em (30), será

$$
\begin{aligned}
b^{*} & =\left[\begin{array}{c}
b_{1}^{*} \\
b_{2}^{*} \\
\vdots \\
b_{M}^{*}
\end{array}\right]=\left[\begin{array}{cccc}
\sigma^{11} X_{1}^{\prime} X_{1} & \sigma^{12} X_{1}^{\prime} X_{2} & \cdots & \sigma^{1 M} X_{1}^{\prime} X_{M} \\
\sigma^{21} X_{2}^{\prime} X_{1} & \sigma^{22} X_{2}^{\prime} X_{2} & \cdots & \sigma^{2 M} X_{2}^{\prime} X_{M} \\
\vdots & \vdots & & \vdots \\
\sigma^{M 1} X_{M}^{\prime} X_{1} & \sigma^{M 2} X_{M}^{\prime} X_{2} & \cdots & \sigma^{M M} X_{M}^{\prime} X_{M}
\end{array}\right]^{-1} \\
& \times\left[\begin{array}{c}
\sum_{u=1}^{M} \sigma^{1 u} X_{1}^{\prime} Y_{u} \\
\vdots \\
\sum_{u=1}^{M} \sigma^{M u} X_{M}^{\prime} Y_{u}
\end{array}\right]
\end{aligned}
$$

e a matriz de variância-covariância do estimador $b^{*}$ é dada por $\left(X^{\prime} \Sigma^{-1} X\right)^{-1}$ ou por:

$$
V\left(b^{*}\right)=\left[\begin{array}{cccc}
\sigma^{11} X_{1}^{\prime} X_{1} & \sigma^{12} X_{1}^{\prime} X_{2} & \cdots & \sigma^{1 M} X_{1}^{\prime} X_{M} \\
\sigma^{21} X_{2}^{\prime} X_{1} & \sigma^{22} X_{2}^{\prime} X_{2} & \cdots & \sigma^{2 M} X_{2}^{\prime} X_{M} \\
\vdots & \vdots & & \vdots \\
\sigma^{M 1} X_{M}^{\prime} X_{1} & \sigma^{M 2} X_{M}^{\prime} X_{2} & \cdots & \sigma^{M M} X_{M}^{\prime} X_{M}
\end{array}\right]
$$

O estimador em (30) possui todas as propriedades de um estimador ótimo, isto é, ele é o melhor estimador linear não-viesado, supondo-se que os dados sejam normais; ele também pode ser considerado um estimador de máxima verossimilhança. Nota-se que a equação (30) é idêntica a um estimador de mínimos quadrados, aplicado equação por equação, quando os distúrbios possuem uma matriz de variância-covariância diagonal, isto é, se $\sigma_{u u^{\prime}}=$ $\sigma_{u^{\prime} u}=0$ para $u^{\prime} \neq u$. Entretanto, quando $\mathrm{X}_{\mathrm{u}}$ é distinto, e os distúrbios em diferentes equações são correlacionados, o estimador em (31) fará diferença na estimação de equação por equação por meio dos mínimos quadrados ordinários.

A aplicação do $S U R$ pode também ser estimada pelo $M M Q O$ em Três Estágios, conforme demonstrado por Zelner \& Theil (1962), pois, dessa forma, é possível a estimação dos parâmetros das equações simultâneas, possibilitando diferentes restrições na estrutura das equações e ganhando-se na eficiência das estimativas.

O método de Três Estágios usa a matriz dos momentos dos distúrbios para estimar todos os coeficientes de todo o sistema, simultaneamente. Dessa forma, o método possui todas as informações, de modo que, se a estrutura de covariância dos distúrbios não for zero, a estimação dos coeficientes, de qualquer equação, ganha em eficiência tão logo outras equações sejam identificadas. Além disso, o método leva em consideração as restrições que são impostas nas diferentes estruturas das equações.

\section{APLICAC̄̃̃O DA METODOLOGIA PROPOSTA}

O controle de realimentação multivariado é necessário quando um conjunto de variáveis será analisado simultaneamente, levando-se em consideração o seu inter-relacionamento. As variáveis consideradas são as temperaturas dos pontos de queima do forno, num total de doze variáveis, formando uma série contendo 92 observações cada, tomadas em intervalos de uma hora, nas três zonas de queima.

Durante a fabricação do azulejo, todas as etapas são importantes, e a cada fase a matéria-prima é testada para verificar se ela atende às especificações da linha de produção. Caso ela não atenda a estas especificações, a matéria-prima é retrabalhada até atingir o valor desejado. A única etapa que não permite esta flexibilidade é a de queima, pois, após ela ser iniciada, não é possível interromper o processo, ou retornar à etapa anterior, devendose esperar que a peça cerâmica complete o ciclo de queima. Um dos maiores problemas, ocorridos em fornos de queima, é a falta de uniformidade da temperatura nas zonas de pré-aquecimento, temperatura máxima e zonas de resfriamento. O forno, em todo o seu comprimento, é dividido em três zonas: na zona de pré-aquecimento a temperatura alcança a faixa de $600^{\circ} \mathrm{C}$; na zona de aquecimento a faixa de $1.000^{\circ} \mathrm{C}$ e, finalmente, na zona de resfriamento, um decréscimo que vai desde $800^{\circ} \mathrm{C}$ até $500^{\circ} \mathrm{C}$.

Como todas as variáveis que compõem o estudo são temperaturas, mas com especificações diferentes, e com controles independentes, elas são denominadas da seguinte maneira. $\mathrm{Na}$ zona de resfriamento tem-se três variáveis RF1, RF2 e RF3; na zona de aquecimento temse as variáveis AQ1, AQ2, AQ3, AQ4, AQ5 e AQ6; e na zona de resfriamento as variáveis RF1, RF2 e RF3. Vale lembrar que existe uma interdependência no efeito conjunto dessas temperaturas.

Segundo Montgomery; Mastrangelo (1991), quando a temperatura é controlada pelo ajuste do posiciona- 
mento de uma válvula, a estatística EWMA pode ser aplicada à série de ajustamentos da válvula, ou, equivalentemente, ao sinal de saída do controlador, que direciona o posicionamento da válvula. E se o algoritmo de ajuste está funcionando adequadamente, problemas que afetem a temperatura irão se refletir nos ajustamentos da válvula. Observa-se que a estatística $E W M A$, no período $t$, é igual a $E W M A$ no período $t-1$, mais uma fração $\lambda$ do erro previsto um passo-à-frente (HUNTER, 1986), e, desse modo, é fácil ver que EWMA é apenas o termo proporcional ao erro.

\section{Modelagem das variáveis selecionadas e determinação dos distúrbios}

A modelagem das variáveis selecionadas será realizada pela metodologia proposta por Zellner (1962). Dessa maneira, será possível o conhecimento dos distúrbios que cada variável irá apresentar.

Os passos, para esse procedimento, são os seguintes:
- estimar as equações, separadamente, utilizando mínimos quadrados;

- utilizar os resíduos de mínimos quadrados, do passo anterior, para estimar as variâncias e covariância dos erros;

- utilizar as estimativas das variâncias dos erros, para estimar as equações conjuntamente.

A variável $R F 1$ foi considerada fora de controle, logo, o conjunto de variáveis que serão modeladas é formado por AQ6, RF1 e RF2, levando-se em consideração as demais variáveis do sistema com uma defasagem inicial de 4 períodos. Dessa maneira, obtém-se não somente o nível de ajuste para a variável identificada, mas o nível de ajuste que deve ser realizado para o conjunto de variáveis. Na Tabela 1, apresentam-se os valores ajustados para o primeiro conjunto de variáveis identificado, os quais foram estimados utilizando-se os programas computacionais PcFiml e PcGive, versão 8.0.

Tabela 1: Estimação do primeiro ponto identificado composto pelas variáveis AQ6, RF1 e RF2

\begin{tabular}{|c|c|c|c|c|}
\hline \multicolumn{5}{|c|}{ MODELAGEM DA VARIÁVEL $\left(A Q G_{T}\right.$} \\
\hline Variáveis & Coeficientes & Erro-padrão & $t$-Student & Significância \\
\hline Constante & 318,19 & 123,11 & 2,585 & 0,0115 \\
\hline$\left(A Q G_{t-1}\right.$ & 0,59543 & 0,080679 & 7,380 & 0,0000 \\
\hline$(R F 2)_{t-1}$ & $-0,20977$ & 0,081842 & $-2,563$ & 0,0122 \\
\hline$(A Q 5)_{t}$ & 0,20646 & 0,052096 & 3,963 & 0,0002 \\
\hline \multicolumn{5}{|c|}{ MODELAGEM DA VARIÁVEL (RF 1) } \\
\hline Variáveis & Coeficientes & Erro-padrão & $t$-Student & Significância \\
\hline Constante & 298,26 & 119,55 & 2,495 & 0,0146 \\
\hline$(R F 1)_{t-1}$ & 0,78693 & 0,051227 & 15,362 & 0,0000 \\
\hline$(A Q 2)_{t-2}$ & $-0,27603$ & 0,097528 & $-2,830$ & 0,0058 \\
\hline$(A Q 5)_{t-1}$ & 0,10266 & 0,047431 & 2,164 & 0,0333 \\
\hline$(P A 2)_{t-1}$ & 0,13931 & 0,054475 & 2,557 & 0,0124 \\
\hline$(P A 1)_{t-2}$ & $-0,13698$ & 0,060355 & $-2,270$ & 0,0258 \\
\hline \multicolumn{5}{|c|}{ MODELAGEM DA VARIÁVEL (RF $1_{T}$} \\
\hline Variáveis & Coeficientes & Erro-padrão & $t$-Student & Significância \\
\hline Constante & 417,77 & 82,171 & 5,084 & 0,0000 \\
\hline$(R F 1)_{t-2}$ & 0,24361 & 0,049103 & 4,961 & 0,0000 \\
\hline$(R F 2)_{t-1}$ & 0,63258 & 0,069200 & 9,145 & 0,0000 \\
\hline$(A Q 1)_{t}$ & $-0,16966$ & 0,051740 & $-3,279$ & 0,0015 \\
\hline$(A Q 2)_{t-2}$ & $-0,16736$ & 0,063493 & $-2,636$ & 0,0100 \\
\hline$(A Q 5)_{t-2}$ & $-0,090479$ & 0,032751 & $-2,763$ & 0,0071 \\
\hline
\end{tabular}


Na Tabela 2 apresentam-se os valores objetivos para cada uma das séries em estudo e os valores previstos para a mesma. Assim, é possível conhecer o distúrbio que cada série apresenta.

Até o presente momento, foi possível determinar o distúrbio que cada variável apresenta, mas ainda é necessário conhecer o nível de ajuste, que deve ser introduzido em cada variável selecionada, o qual será efetuado através do botão controlador da temperatura do forno.

Segundo Sachs et al. (1995), a estrutura de controle, denominada de "Run-by-Run", não é restrita a um modelo de primeira ordem, podendo ser utilizados modelos de ordens superiores, desde que a função do controle mantenha o processo estável.

\section{Aplicação da equação de controle para a realimentação do sistema}

Um estado de controle estatístico implica uma variação aleatória em torno do valor-alvo determinado, produzida por uma ampla variedade de causas comuns. Embora se tenha o esforço de manter o processo próximo do alvo, existem causas como a temperatura ambiente, diferenças em matérias-primas, diferenças entre lotes a serem produzidos, desgastes dos equipamentos ou maquinarias e, até mesmo, diferenças entre operadores, que são de difícil remoção. Nessas circunstâncias, um sistema de regulagem é necessário (BOX, 1991). Até o presente momento foi possível verificar que uma instabilidade afeta o sistema, possibilitando uma futura falta de controle no processo.

Neste item, determina-se o nível de ajuste que deve ser introduzido no sistema, para que a temperatura seja mantida o mais próximo possível do alvo. O sistema é inspecionado e regulado a cada intervalo de uma hora, pois cada alteração introduzida no sistema é realizada no próximo instante, isto é, dentro do período de uma hora, o qual é denominado de sistema responsivo (BOX \& LUCEÑO, 1997).

Para a aplicação da equação de realimentação, ainda será necessário conhecer o valor da constante de ponderação $\lambda$. O método de seleção da constante $\lambda$ é fornecido por Crowder (1989) e Lucas \& Saccucci (1990), sendo que é selecionado aquele que apresenta a melhor performance para o gráfico EWMA, em termos de ARL. Consi- derando os dados correlacionados, Montgomery \& Mastrangelo (1991) sugerem selecionar o valor de $\lambda$, baseando-se na minimização da soma quadrática dos erros.

Nesta etapa, o valor ideal do fator de ponderação será dado pela adequação do ajuste, através da estatística denominada de soma quadrática dos erros. Para a determinação do fator de ponderação $\lambda$, foi feita uma busca com valor inicial para a constante de ponderação de 0,1 , com incremento de 0,01, até o valor máximo de 0,99. Dessa forma, determinou-se o melhor valor para a constante de ponderação que fornece o melhor resultado.

\section{Aplicação do controlador proposto}

A principal idéia de controladores é separar o problema de estimação do problema de controle (ASTROM \& WITTENMARK, 1989). É interessante que um algoritmo de estimação recursiva forneça os parâmetros de estimação para a composição do controlador (DEL CASTILLO, 1996). Conhecidos os valores dos distúrbios de cada variável, que são revelados pela equação de regressão aparentemente não-relacionadas, que leva em consideração a ligação dos erros das equações e os incorpora no modelo e o valor da constante $g$, que determinará o efeito no sistema produtivo, que também é retirado da regressão, sendo o parâmetro da variável que será realimentada, tem-se que encontrar o valor da constante de ponderação $\lambda$.

\section{Equação de controle para o primeiro ponto identificado}

$$
\begin{aligned}
& (A Q 6)_{t}-(A Q 6)_{t-1}=-\frac{0,10}{0,59543}(-3,791)=0,63668 \\
& (R F 1)_{t}-(R F 1)_{t-1}=-\frac{0,1}{0,78693}(3,7813)=-0,48051 \\
& (R F 2)_{t}-(R F 2)_{t-1}=-\frac{0,1}{0,63285}(3,1159)=-0,49236
\end{aligned}
$$

Através dessas equações, é possível conhecer o nível de ajuste que deve ser introduzido em cada variável, para manter o processo o mais próximo do valor-alvo deseja-

Tabela 2: Valores objetivos (valor-alvo), valor previsto e o distúrbio para cada série em estudo.

\begin{tabular}{|c|c|c|c|}
\hline & VALOR OBJETIVO & VALOR PREVISTO & DISTÚRBIO \\
\hline$A Q 6$ & 1030,87 & 1034,661 & $-3,791$ \\
\hline$R F 1$ & 806,37 & 802,5887 & 3,7813 \\
\hline$R F 2$ & 607,859 & 604,7431 & 3,1159 \\
\hline
\end{tabular}


do. Como o botão controlador da temperatura é dotado de uma escala, é possível introduzir um ajuste na variável AQ6, girando o botão em 0,63668 , isto é, em 0,6 unidades no sentido horário, girando 0,5 unidades o botão controlador da temperatura da variável $R F 1$, no sentido antihorário e, finalmente, introduzir um ajuste de 0,5 unidades na variável $R F 2$, girando o botão controlador dessa variável no sentido anti-horário.

Deve-se ressaltar que, neste caso, como o processo está correlacionado à modificação, ou à alteração em uma das variáveis, pode provocar uma alteração nas demais variáveis. Por isso, nesta pesquisa, tem-se a proposta de avaliar e realizar o ajuste de realimentação em um conjunto de variáveis.

A cada hora em que um ajuste for efetuado, uma nova leitura das variáveis é tomada e deve ser analisada, cujo estudo é repetido para verificar se nenhuma alteração ocorreu no sistema. O que se procura, a longo prazo, é a estabilidade do sistema, pois a variabilidade deve diminuir gradativamente, à medida em que as correções forem sendo efetuadas.

A metodologia proposta deverá ser aplicada quando se possuir um conjunto de dados com, no mínimo, três variáveis que se apresentem inter-relacionadas, pois a análise univariada seria deficiente na identificação dos pontos fora de controle, não captando o efeito da correlação entre as variáveis (JACKSON, 1956; APARISI, 1997).

\section{CONCLUSÕES}

O objetivo deste trabalho foi desenvolver uma metodologia auxiliar para a monitoração e/ou realimentação de um sistema multivariado, deixando claras todas as etapas a serem cumpridas, e tornando mais fácil a sua aplicação, fazendo, também, a ligação entre o controle estatístico e o controle de engenharia do processo.

$\mathrm{Na}$ composição do controlador proposto, as constantes de suavização $\lambda$, e de ganho do sistema $g$ foram encontradas de maneira eficiente, tornando o ajuste eficaz, pois, segundo Sachs et al. (1995), as compensações de realimentação freqüentemente são tomadas com base na experiência passada dos profissionais ligados ao processo. Os autores acreditam que o processo de ajuste representa um avanço significativo na tecnologia de processo de controle, o qual reconhece a natureza dos dados e provê uma estrutura para controlá-lo.
Para a obtenção do controlador proposto, após a identificação das variáveis a serem realimentadas, passa-se por distintas fases, até a sua execução final. Essas fases são: a modelagem, a identificação, a estimação, o projeto de controle e a monitoração. Por isso, o sucesso da aplicação do $A C E P$ requer a colaboração não só do profissional encarregado de realizar os ajustes, mas de profissionais com conhecimentos mais específicos em controle de qualidade e séries temporais, para garantir o bom desempenho da metodologia proposta (TUCKER et al., 1993). O conhecimento é importante para a elaboração apropriada dos modelos que representam o processo, para determinar as condições experimentais, estimar parâmetros, validar os resultados, determinar o controle apropriado, implementar o procedimento e monitorá-lo.

Em muitas aplicações práticas, o suporte tecnológico e o conhecimento, às vezes, não estão disponíveis frente a métodos elaborados, mas há a necessidade de se promover este avanço, pois os problemas mais difíceis de serem solucionados necessitam de um conhecimento mais específico, para tornar a empresa mais competitiva. No entanto, o engajamento de todos os profissionais ligados à produção e o apoio gerencial são fundamentais para a implantação dessa metodologia.

A aplicação do $A C E P$, por meio da metodologia proposta, é uma ferramenta muito útil no campo industrial, pois proporciona que o ajuste do processo produtivo seja realizado enquanto as peças, ou produtos, ainda estão na linha de produção. Mas, ao mesmo tempo em que ela é útil, aparece o risco de a metodologia não ser utilizada adequadamente, por falta de conhecimento do profissional. Para isso, sugere-se um treinamento aos colaboradores que estejam envolvidos no processo produtivo, para que a metodologia proposta possa ser adotada com sucesso.

Para uma maior utilização desta pesquisa, seria interessante a aplicação em outros tipos de fornos, os quais possuam as mesmas características que o forno de queima de peças cerâmicas. Também seria útil a sua aplicação em processos multivariados, que não apresentem as características das variáveis em uma seqüência linear, bem como em processos de indústrias de engarrafamento de refrigerantes, por exemplo, que possuem características como pressão, quantidade de gás a ser injetado no vasilhame, volume do líquido que deve ser envasado e que devem ser controlados continuamente.

\section{- Agradecimentos}

Agradecemos à FAPERGS, pelo auxílio financeiro destinado para esta pesquisa; à indústria CECRISA pela cadência dos dados e elucidação das dúvidas em relação ao processo produtivo e, por fim, as sugestões e contribuições oportunas dos revisores. 


\section{Artigo recebido em 18/03/2004 Aprovado para publicação em 27/08/2004}

\section{- Bibliografia}

ASTROM, K.J, and WITTENMARK, B. Adaptative Control. Addison Wesley, Reading, MA. 1989.

APARISI, F. (1997). Sampling plans for the multivariate $\mathrm{T}^{2}$ control chart. Quality Engineering, v.10. n.1, p.141147.

BOX, G.E.P. (1991). Feedback control by manual adjustment. Quality Engineering, 4(1), p. 143-151.

BOX, G.E.P. and JENKINS, G.M. Time series analysis - Forecasting and control. Oakland, CA: Holden-Day, 1970.

BOX, G.E.P; HUNTER, W.G. and HUNTER, J.S. Statistics for experiments. An introduction to design, data analysis and model building. John Wiley \& Sons, Inc. NY. 1978.

BOX, G.E.P.; JENKINS, G.M. and Mac GREGOR, J.F. (1974). Some recent advances in forecasting and control. Applied Statistics, v. 23, p. 158-179.

BOX, G.E.P; JENKINS G.M and REINSEL G.C. Time series analysis: forecasting and control. 3 ed. Prentice Hall Inc. Englewood Cliffs, NJ, 1994.

BOX, G.E.P. and KRAMER, T. (1992). Statistical process control and automated process control - A discussion. Technometrics, August, v. 34 , p. $251-267$

BOX, G.E.P. and LUCEÑO, A. (1997). Discrete proportional-integral adjustment and statistical process control. Journal of Quality Technology, July, v. 29, n. 3.

Statistical control by monitoring and feedback adjustment. John Wiley \& Sons, Inc. NY, 1997.
CHARENZA, W.W. and DEADMAN, D. New directions in econometric practice general to specific modelling, cointegration and vector autoregression. 2 ed. Edward Elgar Publishier Limited, Cheltenham, UK, 1997.

COCHRANE, J.H. Time series for macroeconomics and finance. University of Chicago, Chicago, IL. Spring 1997.

CROWDER, S.V. (1989). Average runs lengths of exponentially weighted moving average control charts. Journal Quality Technology, v. 19, p. 161-164.

CROWDER, S.V, and HAMILTON, M.D. (1992). An EWMA for monitoring a process standard deviation. Journal of Quality Technology, January, v. 24 n. 1, p. 12-21.

DEL CASTIllo, E. (1996). A multivariate self-tuning controller for run-to-run process control under shift and trend disturbances. IIE transactions, v. 28, p. 1011-1021.

ENDERS, W. Applied econometric time series. Wiley series in probability and mathematical statistics. John Wiley and Sons, Inc., New York. N.Y. 1995.

FRANKLIN, F.G.; POWELL, D.J. and EMAMI-NAEINI, A. Feedback control of dynamic systems. 3 ed. AddisonWesley Publishing Company, Inc. New York. N.Y. 1994

HAMILTON, J.D. Time Series analysis Princeton University Press, Princeton - New Jersey, N.J. 1994.

HILL, C.; GRIFFITHS, W.; JUDGE, G. Econometria São Paulo. Editora Saraiva, 1999.

HUNTER, J. S. (1986). The exponentially weighted moving average. Journal of Quality Technology, v. 18 , p. $203-210$
JACKSON, J.E. (1956).Quality control methods for two related variables. Industrial Quality Control, January. p. 4

KEATING, J.W. (1990). Identifying VAR models under rational expectations. Journal of Monetary Economics, v. 25, p. $453-476$

KHURI A.I. \& CONLON M. (1981). Simultaneous optimization of multiple responses represented by polynomial regression functions Technometrics, November. v. $23, \mathrm{n}, 4$.

LUCAS, M.J. and SACCUCCI, M.S (1990). Exponentially weighted moving average control schemes: Properties and enhancements. Technometrics, February. v. 32. n. 1 , p.1-12.

(1990). Exponentially weighted moving average control schemes: Properties and enhancements. Technometrics, v. 20, p. 85-93.

LÜTKEPOHL, H. Introduction to multiple time series analysis. 2. ed. Springer-Verlag Berlin - Germany, 1991.

Mac GREGOR, J.F. (1987). Interface between process control and on line statistical process control. Computational System Technology Division Communication, v. 10, p. 9-20.

MADDALA, G.S. Introduction to econometrics. 2. ed. Prentice-Hall Inc. Englewood Cliffs, New Jersey, 1992.

MONTGOMERY, D.C. and MASTRANGELO, C.M. (1991). Some statistical process control methods for autocorrelated data. Journal of Quality Technology, July, v. 23, n. 3 p.179-204.
MONTGOMERY, D.C; KEATS, J.B.; RUNGER, G.C. and MESSINA, W.S (1994). Integrating statistical process control and engineering process control. Journal of Quality Technology, April, v. 26, n. 2, p. 79-87.

RAMIREZ, W.F. (1994). Process control and identification. Academic Press, Inc. San Diego, C.A.

REINSEL, G. C. Elements of multivariate time series analysis. Springer-Verlag, New York, 1993.

SACHS, E.; HU, A. and INGOLFSSON A. (1995). Run by run process control Combining SPC and feedback control. IEEE Transaction on Semiconductor Manufacturing, February, v. 8, n. 1, p. $26-43$.

SIMS, C.A. (1980). Macroeconomicas and reality. Econometrica 48, p. 1-48.

SHINSKEY, F.G. Feedback controlers for the process industries. Mc Graw Hill, Inc., New York. N.Y. 1994.

TUCKER, W.T; FALTIN, F.W and VANDER WIEL, S.A.V. (1993). Algorithmic statistical process control: An elaboration. Technometrics, November, v. 35. n. 4.

VANDER WIEL, S.A. (1996) Monitoring process that wander using integrated moving average models. Technometrics, May, v. 38, n. 2 .

ZELLNER, A. (1962). An efficient method of estimating seemingly unrelated regressions and tests for aggregation bias. American Statistical Association Journal, June, p. 348- 368 .

ZELLNER, A. and THEIL, H. (1962). Three-stage least squares: Simultaneous estimation of simultaneous equations. Econometrica, January, v. 30, n. 1. 